\title{
Warburg effect: difference in cancer treatment
}

\begin{abstract}
The Warburg effect occurs as a reflected alteration at the mitochondrial level, consisting of the generation of a large amount of lactate and energy in the form of electrons, products of special cellular metabolism, mainly in cancer cells. This paper review focus in how studies in humans, animal models, and cell cultures propose that the warburg effect is an important way to understand the metabolism of the cancer cell and also permit suggest target for anticancer drugs. Studies suggest that in the cancer cell, unlike a normal cell, there is a decoupling between aerobic glycolysis and mitochondrial metabolic processes, due to the hypoxic environment, all this leads to the increased expression of specific enzyme isoforms for cancer, which allows understand the resistance of these cells to restricted environments. The scientific advances regarding how the Warburg effect happens, which proteins and enzymes are involved, give hope in cancer therapeutics, directed to inhibit some of the enzymes that condition it, these can be Akt (Warburg kinase), PK -M2 (the Warburg enzyme), HIF-1- $\alpha$ (the Warburg transcription factor), LDH5. At present the specificity, the selectivity on the cancer cells that can be defined based on the warburg effect, will allow to find new therapeutic targets.
\end{abstract}

Volume 3 Issue I - 2019

\author{
Viola-Rhenals Maricela, Cáceres-Matta \\ Sandra \\ University of Cartagena, Biochemistry and Cell Biology Group, \\ Colombia
}

\begin{abstract}
Correspondence: Viola-Rhenals Maricela, University of Cartagena, Biochemistry and Cell Biology Group, Exacts and Natural Sciences Faculty, Campus San Pablo, Lab 106, Cartagena, Bolivar, Colombia, Tel +57-3017163991,

Emailmviolar@unicartagena.edu.co
\end{abstract}

Received: March 27, 2018 | Published: February 01, 2019

Keywords: warburg effect, cell cancer, anaerobic glycolysis, HIF-1 $\alpha$

Abbreviations: ATP, adenosin triphosphate; $\mathrm{CO}_{2}$, carbon dioxide; DNA, desoxiribonucleotic acid; HIF-1 $\alpha$, hypoxia inducible factor- $\alpha$; HIF- $1 \beta$, hypoxia inducible factor- $\beta$; $\mathrm{LDH}$, lactate dehydrogenase; MnSOD , manganese superoxide dismutase; HRE, hypoxia-responsive element; GLUT, glucose transporter; HK, hexokinase; 1,6BisP, 1,6-bis-phosphate; PEP, phosphoenolpyruvate; $\mathrm{PDH}$, pyruvate dehydrogenase; PFK1, phosphofructokinase1, PHD, proline hydroxylases; ROS, reactive oxygen species; TCA, tricarboxylic acid; $\alpha-\mathrm{KD}, \alpha$-ketoglutarate

\section{Introduction}

Cancer is a disease characterized by the stochastic growth of cells. Cancer cells rewire their metabolism to promote growth, survival, proliferation, and long-term maintenance. Metabolism, the sum of all chemical reactions that take place within a cell or organism, is an area of biochemical research with a wide variety of applications in all scientific fields. Understanding its pathways and regulation is somehow to reveal the cells' function and, ultimately, life itself. Cancer is no exception and a profound knowledge of cancer cell metabolism is invaluable in the comprehension of this pathology. ${ }^{1}$ In eukaryotic cells, cellular respiration is primarily aerobic, but in some instances, cells can produce energy anaerobically through glycolytic fermentation. These two processes share a common initial metabolic pathway called glycolysis which is regulated by phosphofructokinase 1 and which leads to the production of pyruvate from glucose. Under aerobic conditions, pyruvate enters the mitochondria and fuels the tricarboxylic acid cycle which produces citrate, high levels of ATP and $\mathrm{CO}_{2}$. In contrast, under anaerobic conditions, pyruvate remains in the cytosol and is converted into lactate and markedly smaller yield of ATP. ${ }^{2}$ The metabolism of glucose, the central macronutrient, allows for energy to be harnessed in the form of ATP through the oxidation of its carbon bonds. This process is essential for sustaining all mammalian life. In mammals, the end product can be lactate or, upon full oxidation of glucose via respiration in the mitochondria, $\mathrm{CO}_{2}$. In tumors and other proliferating or developing cells, the rate of glucose uptake dramatically increases and lactate is produced, even in the presence of oxygen and fully functioning mitochondria. ${ }^{3}$ The characteristics of these cells is their great resistance to die, being able to live in environments under restrictive conditions within them the most important is the low availability of oxygen. Many efforts are being made to find a therapeutic against tumor cells, however, independently of the stage of the carcinogenic process in which is the tumor at the time of treatment, it is possible the appearance of unwanted side effects, which contribute negatively in the state of the patient and therefore in his recovery. It is therefore necessary to give importance to desired objectives for antitumor chemotherapy such as: a) therapeutic selectivity, proposing that an anticancer drug must be act only in tumor cells and not in normal cells and b) absence of adverse effects produced by therapy due high doses administrated to the patient but to administrate a dose that is effective and not very toxic, to obtain the best balance in the treatment. This balance is difficult to obtain in the real life because at the genetic level both normal and tumor cells are indistinguishable in terms of the superstructure of their hereditary components; while other biochemical processes such as glycolytic metabolism and redox balance may be altered in tumor cells. The common feature of this altered metabolism is increased glucose uptake and fermentation of glucose to lactate. This phenomenon is observed even in the presence of completely functioning mitochondria and together this hypothesis has been studied for many years, giving rise to the theory of Warburg. This aerobic glycolysis (the Warburg effect) facilitates tumour growth and drugs targeting aerobic glycolysis are being developed. The Warburg Effect has been documented for over 90 years and extensively studied over the past years with thousands of papers reporting to have established either its causes or its functions. Otto Warburg first demonstrated that proleferating cancer cells enhanced their glucose consumption and produced lactate, even under aerobic conditions. This reprogrammed cellular metabolism is now recognized as a hallmark of cancer ${ }^{4}$ and it is important to better understand its underlying molecular mechanisms for the development of new therapeutic interventions. ${ }^{3}$ The objective of this article is to conduct a thematic review to examine the studies carried out related to the metabolism of the cancer cell determined by the Warburg effect in order to know possible target for cancer cells.

\section{Historical evolution}

The uncontrolled growth of cancer cells results in a tumor, whose most important feature is the ability of cells to live under conditions 
of low oxygenation or hypoxia. Otto Warburg, chemist who won the Nobel Prize in 1931, made the first investigations related to the cancer cell metabolism in the 1920 s, concluding that in anaerobic conditions the tumor cells have an abnormally decreased glycolytic rate along with a high production of lactate. Based on this observation and taking into consideration the principle of metabolic regulation known as the Pasteur effect, he formulated the hypothesis that a respiratory enzyme, lactate dehydrogenase was involved in the metabolism mainly fermentative for the generation of ATP, ${ }^{5}$ with the subsequent altered function of the mitochondria, all this is the basis of what is now called Warburg Theory.

There are two main metabolic processes by which the cells of mammals obtain energy in the form of ATP: lactic fermentation and aerobic respiration. According to Warburg, the energy efficiency in cancer cells due to lactic fermentation is much higher in aerobic respiration being approximately 15 times greater. ${ }^{5}$ These studies attracted the attention of the scientific community immediately, initiating in vivo experiments related to respiration and fermentation, that is, the metabolism of carbohydrates in tumor cells.

In 1927, O. Warburg public the work "Metabolism of tumors", which describes that cancer is characterized by two basic conditions: acidosis and hypoxia. According to him, tumor cells have a metabolism based on anaerobic glycolysis or fermentation, being more difficult to survive under high oxygen levels; also showed that normal cells can change to cancer cells when they are under hypoxia due to the alteration of the mitochondrial metabolism, which causes the induction of metabolic pathways, some of them compatible with the neoplastic phenomenon. ${ }^{6}$

The neoplastic cells successfully modulate glycolysis, either aerobic or anaerobic, maintaining an energetic profile that allows it to maintain its phenotype, division and metabolic functioning, but without blocking other fundamental routes. Of the total glucose captured by the cell, approximately $5 \%$ sends it to the aerobic route and the remaining $95 \%$ to the anaerobic route, ${ }^{3}$ this justifies the fact that the content of free glucose in the different tissues analyzed, it was low, compatible with a higher production of lactate, all this due to a deviation of respiration towards fermentation. ${ }^{7}$

Among the researchers interested in these findings is Cori who carried out his studies of tumors in the wings of chickens, evidencing an increase in lactic acid, a decrease in free glucose and a $\mathrm{CO}_{2}$ content when analyzing the wing with tumor vs the wing without tumor. These results coincided with Warburg, suggesting that the origin of the cancer could be the damage in the cellular respiration. ${ }^{8}$ The Warburg effect is considered as a characteristic of the majority of the cells of the cancer, originally the anaerobic glycolysis was a universal property of malignant cells, due to defects in mitochondrial respiration. ${ }^{5,9}$

Sauer \& Dauchy ${ }^{10}$ who disagreed for a moment with Warburg, also conducted their investigation with Jensen's sarcoma; they demonstrated that both the production and net use of lactic acid could occur in these tumors, depending on the arterial concentrations of ketone bodies, lactate, and the extent of the fasting condition, being low in contrast to what was reported. It could be suggested that this difference was due to the low specificity of the method used by Warburg to determine the levels of lactic acid (interferences of ketone or acid bodies) and the fact that Jensen's sarcoma was cultured in the peritoneal cavity of the rats, increasing the fast, the production of more ketone bodies.
After more than half a century of research, the Warburg effect is still important to explain the metabolism of cancer cells, however, the exact reasons and physiological values remain contradictory among the investigations, even when the bases remain the same.

During aerobic glycolysis, the production of reactive oxygen species is lower than in anaerobic glycolysis, which results in resistance to tumor apoptosis, this has been identified as one of the defense mechanisms in malignant diseases. ${ }^{11}$ This is why the cause of the Warburg effect has once again attracted the attention of scientists because it allows to differentiate a normal cell from a carcinogen in biochemical terms, so the enzymes involved in glycolysis and the oxidative stress generated, constitute important therapeutic targets in this disease.

\section{Warburg effect}

The Warburg effect can be seen as an alteration of the biochemical metabolism of cancer cells reflected at the energy level. The bases of the effect are due to the fact that due to uncontrolled growth the tumor metabolism must be adapted. For this, the cancer cells adopt anaerobic glycolysis and lactic fermentation as a way to accumulate ATP for their growth under acid and hypoxic conditions. ${ }^{12}$

Warburg investigated and described the changes in the metabolism in cancer cells based on the hypoxic environment, so one of the key proteins is the hypoxia-inducible factor (HIF) that plays an important role in the induction of transcription of isoforms of the glycolytic enzymes, in such a way that the cell can live even in low oxygen concentrations. This hypothesis also involves adaptation to the hypoxic environment in cancer tissues, mitochondrial defects and the abnormal expression of certain metabolic enzymes.

\section{Hipoxia and Warburg}

The glycolytic process of a normal cell occurs in a normoxic environment in which after glucose uptake, it is oxidized for energy. This metabolic pathway consists of 10 consecutive enzymatic reactions that convert glucose into two molecules of pyruvate that will then be directed to the citric acid cycle by means of pyruvate kinase 1 .

In a cancer cell, the glycolytic process occurs in a hypoxic environment, HIF being an important factor for the resistance of these cells, in which $95 \%$ of the energy is obtained by means of anaerobic glycolysis and only $5 \%$ through the aerobic route, due to the intervention of HIF-1.

The hypoxia-1-inducible transcription factor (HIF-1) has two isoforms HIF- $1 \alpha$ and HIF- $1 \beta$, the first one producing the changes in cancer cells. Under conditions of normoxia, HIF-1 $\alpha$ is degraded by hydroxylation catalyzed by proline hydroxylases (PHDs), an enzyme of the $\alpha$-ketoglutarate family ( $\alpha-\mathrm{KG}$ ) -dependent. This hydroxylation in proline 402 and 564 directs it for ubiquitination. ${ }^{13}$ Under hypoxic conditions, the PHD is inactive, HIF- $1 \alpha$ does not degrade and migrates to the nucleus where it binds to HIF-1 $\beta$, the cofactor p300 and DNA polymerase II that bind to the hypoxia-responsive element (HRE) of the target genes.

\section{Glycolytic metabolism according to Warburg}

The first identified biochemical characteristic of tumor cells is the change in glucose metabolism and oxidative phosphorylation. One of the most important mitochondrial functions is the production of energy in the form of ATP. In normal cells most of the ATP is produced in the 
mitochondria through the process called oxidative phosphorylation, although various substrates can serve as fuel for producing energy; the main fuel is glucose for most cells.

Glycolysis is the metabolic pathway responsible for oxidizing glucose in order to obtain energy for the cell. It consists of consecutive enzymatic reactions that convert glucose into two pyruvate molecules and then direct it to the Krebs cycle or citric acid cycle. In cancer cells the key to the process of glycolysis is HIF-1, as already mentioned consists of two subunits $\alpha-\beta$, highlighting the subunit HIF-1a that is induced only in a hypoxia state. In the cancer cells the oxygen levels are diminished which affects the activity of the PHD2 which is an oxygen sensor and in the state of hypoxia it is inactivated due to this the HIF- $1 \alpha$ escapes from its degradation and migrates to the cell nucleus where it is initiates the transcription of genes that promote the expression of specific membrane transporters such as GLUT1 and GLUT3, isoenzymes that intervene in the 10 consecutive reactions that convert glucose to 2 pyruvate molecules such as hexokinase 2 , phosphofructokinase 2 , pyruvate kinase 2 and lactate dehydrogenase- 5 which is responsible for directing the two pyruvate molecules to the lactate pathway. ${ }^{14}$

HIF, as in glycolysis in the mitochondria, induces the activation of isoenzymes of SDH and FH. SHD catalyzes the conversion of succinate to fumarate in the tricarboxylic acid (TCA) cycle reactions, with the release of a flavin-adenine dinucleotide molecule. SDH is composed of four subunits: SDHA, SDHB, SDHC and SDHD, which are the classic SDH complex components. In recent years a new factor was identified as a member of the SDH complex and was named as $\mathrm{SDH} 5$, which is a participant in SDHA flavinacion. ${ }^{15} \mathrm{SDH}$ mutations are commonly found in paraganglioma, gastric stromal tumors, and acute T-cell leukemia in children. The detected mutations of SDH in cancer cells are: SDHB, SDHC, SDHD and SDH5 but not SDHA. ${ }^{16}$

In the SDH and FH mutations in tumors, HIF1- $\alpha$ and its amplification target genes are activated, it is hypothesized that mutations in SDH and FH induce the tumorogenicity through the activation of the HIF1- $\alpha$ pathway. We found mutations of SDH and FH with accumulation of succinate and fumarate, structural analogs of $\alpha-K G$ that can inhibit PHDs and activate the HIF-1 pathway. ${ }^{17}$

\section{GLUT I and GLUT3 membrane transporter}

Glucose is transported to the intracellular medium mainly through glucose transporters (GLUT), which comprises six isoforms that differ in the specificity for hexose and tissue localization among other variables; it follows that each cell captures this carbohydrate differently, influencing both glycolysis and glycogen genesis. The expression of GLUT1 and GLUT3 mediated by HIF-1 $\alpha$. A high expression of these two isoforms has been associated with the poor prognosis of various human tumors such as breast cancer and prostate cancer. $^{18}$

\section{Glycolytic enzymes}

Some enzymes are important because are altered in Warburg effect, and them are possible target as antitumoral.

Hexokinase 2: It is a member of the HK family, which is the first enzyme to participate in the process of glycolysis when glucose enters the cell; phosphorylating it to glucose-6P hexokinase- 2 is expressed by means of factor HIF-1 in a hypoxia state. Like GLUT1 and GLUT3, high concentrations of hexokinase 2 are of poor prognosis for different types of cancer in humans. ${ }^{19}$
Fosfofructokinase 2: Is one of the enzymes responsible for the regulation of the speed of glycolysis. Involved in the conversion of fructose 6P to fructose 1,6BisP. The control of the activity of phosphofructokinase- 2 is related to the functioning of the heart and the hypoxic state in the cancer cell. ${ }^{20}$

Pyruvatokinase 2: Induces the dephosphorylation of phosphoenolpyruvate (PEP) in pyruvate for the production of ATP, there are four isoforms laPKM1 / M1-PK and PKM2 / M2-PK are the two isoforms that are expressed in higher concentration in cancer cells by medium of the HIF. High concentrations of this isoenzyme is related to glycolysis and tumor angiogenesis. ${ }^{21}$

Lactate dehydrogenase 5: Is the enzyme that directs $95 \%$ pyruvate to anaerobic glycolysis or lactate pathway. There are five isoforms of LDH-1, LDH2, LDH3, LDH4 and LDH5. Elevated levels of type LDH4 and LDH5 are associated with aggressiveness and poor tumor prognosis. ${ }^{22}$

Pyruvate dehydrogenase kinase 1: It is the enzyme that directs $5 \%$ of pyruvate produced in the glycolysis of the cancer cell to the Krebs cycle. Research has reported that high concentrations of PDK1 is associated with head and neck cancer. ${ }^{23}$

The induced proteins include all the differential enzymes expressed in cancer and not in normal cells, allowing these cells the behavior of obtaining energy for uncontrolled growth. The regulation of PDK is able to inhibit the enzymatic activity of pyruvate dehydrogenase $(\mathrm{PDH})$, and the increase in LDH-A levels can accelerate the conversion of pyruvate to lactate. The target proteins produced by the activation of transcription by HIF- $1 \alpha$ synergistically promote the Warburg effect. ${ }^{24}$

\section{Mitochondria and warburg effect}

The understanding of the Warburg effect, has allowed to justify the non-involvement of the mitochondria in obtaining energy by a cancer cell, even if it explains the oxidative stress observed in these cells.

Oxidative stress is a condition of all cells that occurs due to an imbalance between the reactive oxygen species (ROS) that are produced in the mitochondria and the antioxidant defense mechanisms. In the absence of oxygen, the cell does not have an electron acceptor remaining free in the mitochondria producing this cellular event. In cancer, its cells are resistant to high concentrations of these compounds, and even induce signaling pathways to evade apoptosis and induce growth, so some are considered markers of poor prognosis as is for example the enzyme manganese superoxide dismutase (MnSOD).

In the metabolism for the production of ATP the eukaryotic cell does it through the process of oxidative phosphorylation, and as a byproduct of aerobic glycolysis, ROS is generated, this occurs due to the incomplete reduction of oxygen; the electrons pass through the electron transport chain. The production of superoxide anion $\left(\mathrm{O}^{2-}\right)$, is produced by the transfer of a single electron to the oxygen molecule during oxidative phosphorylation. It has been estimated that under physiological conditions $1 \%$ to $2 \%$ of molecular oxygen becomes ROS. ${ }^{25}$

The inhibition of ROS production can be a valuable approach to the prevention and treatment of cancer. Treatment based on an antioxidant diet has been shown to inhibit the growth of tumors in animals. In fact, there is evidence that dietary antioxidants taken concurrently with conventional cancer treatment may actually do harm by protecting cancer cells from the pro-oxidant effects of chemo and / or 
radiotherapy. ${ }^{26}$ The development of antioxidants specifically targeting the mitochondria may be the hope to fight cancer. As mentioned before, high levels of ROS are toxic to cells and lead to death by apoptosis or necrosis. Since cancer cells generally have higher ROS levels than normal cells, which are in higher stress conditions and are more sensitive to death by the promotion of ROS agents. In effect the therapeutic agents against cancer such as; Cisplatin, vinblastine, doxorubicin, ionizing radiation among others, exert their action through ROS in cell death. Numerous therapies designed to promote the overproduction of ROS are currently under investigation for the selective death of cancer cells. ${ }^{25}$

\section{Conclusion}

Many are the investigations that relate Warburg's theory to the metabolism of cancer cells. They describe that the change in aerobic glycolysis metabolism is a common hallmark in cancer cells, they seem to have adapted in order to facilitate the incorporation of nutrients for the formation of new cells. Although much remains to be learned from regulating the metabolism of cancer cells, it is increasingly clear that metabolic reprogramming is induced by HIF-1, PKM2, IDH, SH, GLS and other molecules that act independently or with each other. Cancer-specific metabolites have recently been used for the diagnosis and therapeutics of cancer.

With regard to this subject after the Warburg studies from the 20 s to $50 \mathrm{~s}$, there was a stagnation and it was already in the $80 \mathrm{~s}$ that it resumed being booming at this time, due to the involvement it has in the search for new targets therapeutic, specific and selective for tumor cells, for these e constitutes an important field of research.

\section{Acknowledgments}

None.

\section{Conflicts of interest}

The authors declare there is no conflicts of interest.

\section{References}

1. Ferreira L. Cancer metabolism: The Warburg effect today. Exp Mol Pathol. 2010;89(3):372-380.

2. Icard P, Shulman S, Farh D, et al. How the Warburg effect supports aggressiveness and drug resistance of cancer cells? Drug Resist Updat. 2018;38:1-11.

3. Liberti MV, Locasale JW. The warburg effect: how does it benefit cancer cells? Trends Biochem Sci. 2016;41(3):211-218.

4. Hanahan D, Weinberg RA. Hallmark of cancer: the next generation. Cell. 2011;144(5):646-674.

5. Warburg $\mathrm{O}$. On the origin of cancer cells. Science. 1956;123(3191):309-314.

6. Warburg O, Wind F, Negelein E. The metabolism of tumors in the body. J Gen Physiol. 1927;8(6):519-530.

7. Vander Heiden MG, Cantley LC, Thompson CB. Understanding the Warburg effect: the metabolic requirements of cell proliferation. Science. 2009;324(5930):1029-1033.

8. Cori CF, Cori GT. The carbohydrate metabolism of tumors. I. The free sugar, lactic acid, and glycogen content of malignant tumors. $J$ Biol Chem. 1925;64:11-22.
9. Gogvadze V, Orrenius S, Zhivotovsky B. Mitochondria in cancer cells: what is so special about them? Trends Cell Biol. 2008;18(4):165-173.

10. Sauer LA, Dauchy RT. In vivo lactate production and utilization by Jensen sarcoma and Morris hepatoma 7288CTC. Cancer Res. 1986;46(2):689-693.

11. Altenberg B, Greulich KO. Genes of glycolysis are ubiquitously overexpressed in 24 cancer classes. Genomics. 2004;84(6):1014 1020.

12. Clausen SW. A method for the determination of small amounts of lactic acid. $J$ Biol Chem. 1922;52:263-280.

13. Ema M, Hirota $\mathrm{K}$, Mimura $\mathrm{J}$, et al. Molecular mechanisms of transcription activation by HLF and HIF1-alpha in response to hypoxia: their stabilization and redox signal induced interaction with CBP/p300. EMBO J. 1999;18(7):1905-1914.

14. Semenza GL. HIF-1: upstream and downstream of cancer metabolism. Curr Opin Genet Dev. 2010;20(1):51-56.

15. King A, Selak MA, Gottlieb E. Succinate dehydrogenase and fumarate hydratase: linking mitochondrial dysfunction and cancer. Oncogene. 2006;25(34):4675-4682.

16. Baysal BE, Willett-Brozick JE, Lawrence EC, et al. Prevalence of SDHB, SDHC, and SDHD germline mutations in clinic patients with head and neck paragangliomas. J Med Genet. 2002;39(3):178-183.

17. Hao HX, Khalimonchuk O, Schraders M, et al. SDH5, a gene required for flavination of succinate dehydrogenase, is mutated in paraganglioma. Science. 2009;325(5944):1139-1142.

18. Gould GW, Thomas HM, Jess TJ, et al. Expression of human glucose transporters in Xenopusoocytes: kinetic characterization and substrate specificities of the erythrocyte, liver and brain isoforms. Biochemistry. 1999;30(21):5139-5145.

19. Mathupala SP, Rempel A, Pedersen PL. Glucose catabolism in cancer cells: identification and characterization of a marked activation response of the type II hexokinase gene to hypoxic conditions. $J$ Biol Chem. 2001;276(46):43407-43412.

20. Okar DA, Lange AJ. Fructose-2,6-bisphosphate and control of carbohydrate metabolism in eukaryotes. Biofactors. 1999;10(1):114.

21. Mazurek S. Pyruvate kinase type M2: a key regulator of the metabolic budget system in tumor cells. Int J Biochem Cell Biol. 2011;43(7):969-980.

22. Koukourakis MI, Giatromanolaki A, Sivridis E, et al. Lactate dehydrogenase 5 (LDH- 5) overexpression in non-small-cell lung cancer tissues is linked to tumour hypoxia. Angiogenic factor production and poor prognosis. Br J Cancer. 2003;89(5):877-885.

23. Wigfield SM, Winter SC, Giatro manolaki A, et al. PDK-1 regulates lactate production in hypoxia and is associated with poor prognosis in head and neck squamous cancer. Br J Cancer. 2008;98(12):19751984.

24. Gordan JD, Thompson CB, Simon MC. HIF and c-Myc: sibling rivals for control of cancer cell metabolism and proliferation. Cancer Cell. 2007;12(2):108-113.

25. Ott M, Gogvadze V, Orrenius S, et al. Mitochondria, oxidative stress and cell death. Apoptosis. 2007;12(5):913-922.

26. Han D, Antunes F, Canali R, et al. Voltage-dependent anion channels control the release of the superoxide anion from mitochondria to cytosol. J Biol Chem. 2003;278(8):5557-5563.

27. Fulda S, Galluzzi L, Kroemer G. Targeting mitochondria for cancer therapy. Nat Rev Drug Discov. 2010;9(6):447-464. 